\title{
Dachengqi Decoction Attenuates Intestinal Vascular Endothelial Injury in Severe Acute Pancreatitis in Vitro and in Vivo
}

\author{
Li-yun Pan ${ }^{\mathrm{a}} \quad$ Ya-feng Chen ${ }^{\mathrm{a}}$ Hong-chang Li ${ }^{\mathrm{a}}$ Li-ming Bi ${ }^{\mathrm{a}}$ Wen-jie Sun ${ }^{\mathrm{a}}$ \\ Gui-fang Sun ${ }^{\mathrm{a}}$ Xiao-fen Zhang ${ }^{\mathrm{a}} \quad \mathrm{Ke} \mathrm{Xu}^{\mathrm{b}}$ Dian-xu Feng ${ }^{\mathrm{a}}$ \\ aDepartment of general surgery, Putuo Hospital, Shanghai University of Traditional Chinese Medicine, \\ Shanghai, 'Laboratory Center, Putuo Hospital, Shanghai University of Traditional Chinese Medicine, \\ Shanghai, China
}

\section{Key Words}

Acute necrotizing pancreatitis - Dachengqi decoction - Aquaporin 1 - Junctional adhesion molecule-C - Matrix metalloproteinase $9 \cdot$ Angiopoietin-1 - Endothelial cells • Capillary permeability

\begin{abstract}
Background/Aims: Dachengqi decoction (DCQD) is a well-known traditional Chinese herbal drug with strong anti-inflammatory effects. Angiopoietin-1 (Ang-1) plays a vital role in maintaining the stability and integrity of the vascular wall and prevents vascular leakage due to inflammatory mediators. Our previous work found that DCQD protects against pancreatic injury in rats with severe acute pancreatitis (SAP). This study aims to investigate the effects of DCQD on intestinal endothelial damage in both damaged human umbilical vein endothelial cells (HUVECs) and SAP rats. Methods: HUVECs were randomly divided into four groups: control group, TNF- $\alpha$ group, TNF- $\alpha$ plus Ang-1 group (Ang-1 group), and TNF- $\alpha$ plus DCQD group (DCQD group). Cells were incubated for $6 h, 12 h$, and $24 h$, before collection. The treatment concentration of DCQD was decided based on a Cell Counting Kit-8 (CCK-8) assay. The monolayer permeability of the HUVECs was assessed by measuring the transendothelial electrical resistance (TEER). Apoptosis was analyzed by flow cytometry. mRNA and protein expression of aquaporin 1 (AQP-1), matrix metalloproteinase 9 (MMP9), and junctional adhesion molecule-C (JAM-C) was evaluated by RT-PCR, immunocytofluorescence, and western blot. Forty male Sprague-Dawley rats were randomized into a control group, SAP group, SAP plus Ang-1 group (Ang-1 group), and SAP plus DCQD group (DCQD group). SAP was induced by intraperitoneal injection of cerulein and lipopolysaccharide (LPS), while the control group received $0.9 \%$ saline solution. Evans blue was injected through the penile vein and the rats were then sacrificed $12 \mathrm{~h}$ after modeling. Levels of serum amylase, TNF- $\alpha, \mathrm{IL}-1 \beta, \mathrm{IL}-2$, and IL- 6 were determined by using ELISA. Intestinal tissue was analysed by histology, and capillary permeability in the tissues was evaluated by Evans blue extravasation assay. Protein and

L. Pan and Y. Chen contributed equally to this work.

Dian-xu Feng

and $\mathrm{Ke} \mathrm{Xu}$

\section{KARGER}


mRNA expression of AQP-1, MMP9, and JAM-C were assessed by immunohistofluorescence, western blot, and RT-PCR. Results: DCQD reduced the permeability of HUVEC induced by TNF- $\alpha$ in vitro. Furthermore, DCQD altered the mRNA and protein levels of JAM-C, MMP9, and AQP-1 in HUVECs after TNF- $\alpha$ induction. SAP intestinal injury induced by cerulein combined with lipopolysaccharides was concomitant with increased expression of JAM-C and MMP9, and reduced expression of AQP-1 in intestinal tissue. Pretreatment with DCQD attenuated SAP intestinal injury and lowered the levels of serum amylase, TNF- $\alpha$, IL- $1 \beta$, IL- 2 , and IL- 6 effectively. Our study demonstrated that DCQD decreased the expression of JAM-C and MMP9 and increased the expression of AQP-1 both in vitro and in vivo. Conclusion: DCQD can reduce capillary endothelial damage in acute pancreatitis-associated intestinal injury and the mechanism may be associated with the regulation of endothelial barrier function-associated proteins AQP-1, MMP9, and JAM-C.

\section{Introduction}

Severe acute pancreatitis (SAP) is a common and potentially lethal acute inflammatory disease with no available molecular targeted therapy for the disorder. SAP is due to trypsinogen activation, which leads to inflammatory response including autodigestion, edema, hemorrhage, and even necrosis of the pancreas [1-3]. The pathogenic mechanism of SAP has not been fully investigated whatever the cause. SAP eventually induces regional and systematic inflammatory responses, as well as excessive production of pro-inflammatory cytokines and a waterfall-like cascade reaction triggered by cytokines. Uncontrolled inflammatory response usually results in systemic inflammatory response syndrome (SIRS) and multiple organ dysfunction syndrome, both of which contribute to the high mortality rate of SAP $[4,5]$. Therefore, new treatments or drugs to control the release of inflammatory factors are urgently needed.

The intestine is a major organ involved in the extra-pancreatic stress response in SAP, is extremely vulnerable to inflammatory factors, and correlates strongly with the evolution of the disease [6, 7]. Intestinal microcirculatory disorder and a reduction in blood flow cause an increase in the permeability of the intestinal mucosa and damage the intestinal barrier, which results in the translocation of intestinal endotoxins, bacteria, and other substances into the circulation, thus exacerbating the disease. The mechanism of damage mainly consists of two parts: dysfunction of the intestinal mucosal epithelial barrier, including the mechanical, chemical, immune, and biological barriers, and dysfunction of the intestinal capillary endothelial barrier [8].

The capillary endothelial barrier is a semi-selective barrier composed of a monolayer of endothelial cells around the vascular lumen and basement membrane. Capillary permeability is governed by three factors: the endothelial cells, endothelial intercellular connection, and basement membrane. Aquaporins (AQPs), matrix metalloproteinases (MMPs), and junctional adhesion molecules (JAMs) are important structural proteins of these parts, respectively. Studies showed that aquaporin 1 (AQP-1), matrix metalloproteinase 9 (MMP9), and junctional adhesion molecule-C (JAM-C) all play key roles in regulating capillary permeability in SAP [9-11]. AQP-1 is crucial to the translocation and balance of water both inside and outside the cells and blood vessels. Following stimulation by inflammatory factors, MMP9 can up-regulate and destroy the basement membrane, thus, it plays an important role in microvascular permeability. JAM-C is an important protein at the intercellular junction, and if stimulated by inflammation will localize at the cellular junctions and increase intercellular permeability. These endothelial barrier function-related proteins play critical roles in the regulation of capillary permeability in SAP.

Angiopoietin-1 (Ang-1) is the only endogenous protein factor that can resist the damage of vascular leakage among the angiogenic factor family. Our previous studies found that serum Ang- 1 in rats with SAP is significantly decreased and after Ang- 1 treatment, the inflammatory factors in serum decline dramatically and the capillary permeability of the 
tissue decreases. Therefore, we used Ang-1 in the positive control group.

Dachengqi Decoction (DCQD), recorded in the Treatise on Febrile and Miscellaneous Diseases, is the strongest purgative Sanchengqi Decoction (comprising three different but similar decoctions in a series of Chengqi decoctions) established 2000 years ago by Zhang Zhongjing of the Han dynasty. Combinations of traditional Chinese and Western treatments are more comprehensive and effective for relieving symptoms than western medicine alone by controlling the course of the disease and reducing mortality [12]. Many studies have investigated the mechanisms of action of various decoctions for the treatment of SAP [13, 14]. Our previous studies found that DCQD significantly improves pathological pancreatic damage in a mouse model of acute necrotizing pancreatitis, inhibits inflammatory reactions, alleviates capillary leakage, and eases the symptoms of SAP-complicated SIRS [15]. These findings may provide some guidance for the clinical treatment of SAP. However, the protective effect of capillary permeability and the mechanism in SAP associated with intestinal injury has not been reported.

In this study, using in vivo and in vitro models, we aimed to observe the therapeutic effects of DCQD on SAP-complicated intestinal injury and discuss the specific DCQDmediated mechanism that reduces intestinal capillary permeability from the perspective of the endothelial barrier. SAP rats in vivo were used to observe the effect of DCQD on tissue capillary leakage in vivo, and TNF- $\alpha$ damaged human umbilical vein endothelial cells (HUVECs) aimed to observe the direct effects of DCQD on endothelial cells in vitro.

\section{Materials and Methods}

\section{Drugs and reagents}

DCQD is composed of Da Huang (Radix et Rhizoma Rhei), Houpu (Cortex Magnoliae Officinalis), Zhi Shi (Fructus Aurantii Immaturus) and Mangxiao (Natrii Sulphas) (Shanghai Hua Yu Chinese Herbs Co, Lt, China). Dahuang (1200 g), Mangxiao (750 g), Zhishi (990 g), and Ноирu (900 g) were pulverized and then percolated in $60 \%$ ethanol for $24 \mathrm{~h}$. Thereafter, $3 \mathrm{~L}$ of the percolated liquid was harvested at $1-3 \mathrm{~mL} / \mathrm{min}$ and an additional $60 \%$ ethanol was added to provide full extraction of the active reagent. Mangxiao was ground and filtered through an 80 mesh sieve. Zhishi and Houpu were boiled twice, first for $2 \mathrm{~h}$ and then for a further $1.5 \mathrm{~h}$, harvested, filtered, concentrated, and mixed with the Dahuang percolate. Mangxiao was mixed with ethanol distilled under reduced pressure, filtered by a 10 mesh sieve, and dried to powder. The extraction rate was $13.4 \%$.

Reagents were purchased as follows: cerulein, LPS, trypsin inhibitor (Sigma-Aldrich, St. Louis, MO); Recombinant Human TNF- $\alpha$ (Peprotech Inc., Rocky Hill, NJ); Recombinant Human Angiopoietin-1 (R\&D Systems, Inc., NE Minneapolis, MN); COMP (rt): Angiopoietin-1 (ENZO Life Sciences, Farmingdale, NY); Hank's Balanced Salt Solution (Gibco Industries Inc., Big Cabin, OK); Goat-anti-rabbit antibody, Rabbit-antigoat antibody (Cell Signaling Technology Inc., Danvers, MA); ECL Western Blotting Substrate (Millipore, Billerica, MA); Immunohistofluorescence Kit (Boster, Wuhan, China); Fluorescein isothiocyanate (FITC) Annexin V Apoptosis Detection Kit I (BD Biosciences Pharmingen, San Jose, CA); TRIzol reagent (Invitrogen, Carlsbad, CA); ReverTra Ace qPCR RT Kit (Toyobo Co, Ltd. Life Sciences Department, Osaka, Japan).

\section{Materials}

Cellular model

HUVECs (Shanghai Institutes for Biological Sciences, Shanghai, China) were cultured in DMEM containing 10\% fetal bovine serum (FBS) and 1\% penicillin-streptomycin (P/S, Life Technologies Corp., Grand Island, NY) All cells were incubated at $37{ }^{\circ} \mathrm{C}$ in $5 \% \mathrm{CO}_{2}$ (v/v). TNF- $\alpha$-damaged HUVEC model was established by culture with $10 \mathrm{ng} / \mathrm{mL}$ TNF- $\alpha$ [16] for $6 \mathrm{~h}, 12 \mathrm{~h}$ or $24 \mathrm{~h}$. Cells were divided into 4 groups: control, TNF- $\alpha$ (10 ng/mL TNF- $\alpha$ ), Ang-1 (100 ng/mL Ang-1 30 min after TNF- $\alpha$; Pro Pack Technologies Pvt. Ltd., Shanghai, China), and DCQD groups (100 $\mu \mathrm{g} / \mathrm{mL} 30 \mathrm{~min}$ after TNF- $\alpha)$.

\section{Animal model}

This research was approved by the Ethics Committee of Shanghai Putuo Central Hospital, which is affiliated with the Shanghai University of Traditional Chinese Medicine. Healthy male Sprague-Dawley (SD) 
rats ( $\mathrm{n}=40$; weight $250 \pm 15$ g) were purchased from SLAC Laboratory Animal Co., Ltd (Shanghai, China) and raised in a specific-pathogen-free (SPF) animal room. One week after adaptive feeding, the rats were allocated to either the control group, acute pancreatitis group (SAP group), Ang-1 combined with SAP group (Ang-1 group) (20 ng/kg, ENZO Life Sciences), or DCQD combined with SAP group (DCQD group, $0.9 \mathrm{~g} / \mathrm{kg}$ gavage). The SAP model was induced by 4 abdominal injections of cerulein $(50 \mu \mathrm{g} / \mathrm{kg} / \mathrm{h})$ and 2 abdominal injections of lipopolysaccharides (LPS, $7.5 \mathrm{mg} / \mathrm{kg}$ ), while $0.9 \%$ saline solution was used in the control group. Evans blue (EB) was injected through the penile vein and then the rats were sacrificed $12 \mathrm{~h}$ after the last abdominal injection. Abdominal aortic blood was drawn and immediately centrifuged (1000 g for $5 \mathrm{~min}$ ). The supernatant was extracted and frozen at $-80^{\circ} \mathrm{C}$. Intestines were harvested, and fixed with $10 \%$ formalin for pathology and immunohistochemistry. The remaining parts were frozen at $-80^{\circ} \mathrm{Cuntil}$ needed for the protein assays.

\section{Cell Counting Kit-8 (CCK-8) assay}

A total of 10000 HUVECs were plated into each well of a 96-well plate and cultured for $6 \mathrm{~h}, 12 \mathrm{~h}$, or 24 h. Thereafter, $0.001,0.01,0.1,1,10,100$, and $1000 \mu \mathrm{g} / \mathrm{mL}$ DCQD $(1000 \mu \mathrm{g} / \mathrm{mL})$ was added to the culture medium, and cells were cultured for 6 h, 12 h, and 24 h. Subsequently, $10 \mu \mathrm{L}$ of Cell Counting Kit-8 solution was added to each well and the cells were incubated for $3 \mathrm{~h}$; a microplate reader was used to measure absorbance at $450 \mathrm{~nm}$.

Serum amylase, IL-1 $\beta, I L-2, I L-6$, and TNF- $\alpha$ assays

The serum amylase assay was performed in an automatic biochemistry analyzer (Roche Ltd., Basel, Switzerland). IL-1 $\beta$, IL-2, IL-6, and TNF- $\alpha$ levels were measured by enzyme-linked immunosorbent assay in accordance with the manufacturer's instructions (Hangzhou Multi Sciences, Biotech, Co., Ltd., Gongshu, China).

\section{Pathological changes in the intestine}

Small pieces of intestinal tissue were used for histological analysis. Samples were fixed with $10 \%$ formalin, dehydrated, and embedded in paraffin wax before slicing and staining with hematoxylin \& eosin. The pathological injury severity score was determined under light microscopy according to the method by Chiu et al [17]: 0 indicates normal small intestine mucosa villi; 1 indicates Gruenhagen's space beneath the intestinal mucosal epithelium at the apex of the villus, which often accompanies capillary hyperemia; 2 indicates intestinal mucosa epithelial cells rising from the intrinsic membrane and expansion of the spaces under the intestinal epithelium; 3 indicates most of the intestinal mucosa epithelium is raised, villi are toppled over on both sides, and the top parts of the villi have fallen off; 4 indicates scaling and peeling of the intrinsic membrane, expansion of bare capillaries, and intrinsic membrane components increase; and 5 indicates digestion or metamorphosis of the lamina propria, bleeding, or ulceration.

\section{Capillary permeability test}

The rats were injected with EB (25 mg/kg body weight) via the jugular vein 30 min before they were sacrificed. The intestines were harvested and washed with PBS containing $5 \mathrm{mmol} / \mathrm{L}$ ethylene diamine tetraacetic acid to remove residual blood. The intestinal tissues were weighed and mixed with dimethylformamide at $100 \mathrm{~mL} / 100 \mathrm{mg}$ of tissue followed by incubation for $18 \mathrm{~h}$ at $60{ }^{\circ} \mathrm{C}$. The extracts were centrifuged at $1000 \mathrm{~g}$ for $20 \mathrm{~min}$. The absorbance of the supernatant was then measured by spectrophotometry at $620 \mathrm{~nm}$, and the results were expressed in units of $\mu \mathrm{g} \mathrm{EB} / \mathrm{g}$ intestine, calculated against a standard curve [18].

\section{Transendothelial electrical resistance assay}

The transendothelial electrical resistance (TEER) of the HUVEC monolayers was determined using MERSSTx01 Electrode according to the manufacturer's instruction manual (EMD Millipore Corporation, Billerica, MA). A total of $1 \times 10^{5}$ HUVECs were seeded on each fibronectin-coated plates; blank filters were stratified with fibronectin. Transwell ${ }^{\circledR}$ filters $(0.4 \mu \mathrm{m})$ were used until confluence. After cell culture for $24 \mathrm{~h}$, a MERSSTx01 electrode was used to detect the TEER values of the HUVECs. The resistance values of all Transwell filters were measured sequentially and the mean was expressed in units of $\mathrm{V} \mathrm{cm}{ }^{2}$ after subtraction of the value of a blank cell-free filter. 


\section{Cellular Physiology Cell Physiol Biochem 2017;44:2395-2406

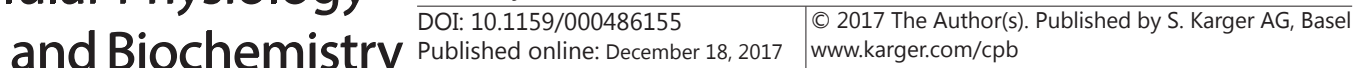

Pan et al.: Dachengqi Decoction Ameliorates Intestinal Injury in Experimental Acute

Pancreatitis

\section{Apoptosis assay}

For the apoptosis assays, cells were plated in 6 -well plates at a density of $3.0 \times 10^{5}$ cells per well. The cells were incubated until they were approximately $80 \%$ confluent, and the assay medium was added. Cells were pretreated with or without TNF- $\alpha(10 \mathrm{ng} / \mathrm{mL})$, then treated with Ang-1 (100 ng/mL) or DCQD (100 $\mu \mathrm{g} / \mathrm{mL}$ ) for $6 \mathrm{~h}, 12$, h or $24 \mathrm{~h}$. Apoptosis rates were determined by the FITC Annexin V Apoptosis Detection Kit I (BD Biosciences Pharmingen) and analysis by flow cytometry was performed within $1 \mathrm{~h}$.

\section{Immunofluorescence}

Fresh intestinal tissues were sectioned and frozen before incubation in 5\% FBS for 30 min. The following antibodies were added and incubated overnight: JAM-C (1:50; Santa Cruz Biotechnology Inc., Santa Cruz, CA), AQP-1 (1:250; Abcam, Carlsbad, CA), and MMP9 (1:250; Abcam). The secondary antibody (Cy3-AffiniPure Goat Anti-Rabbit IgG, Jackson ImmunoResearch, West Grove, PA,) was added and incubated at room temperature for $1 \mathrm{~h}$. Nuclei were stained by DAPI and sections were sealed by glycerinum. Observations were performed at 40x magnification.

Four groups of HUVECs were stimulated for $12 \mathrm{~h}$. Cells were washed with PBS and fixed in $3.7 \%$ paraformaldehyde for $10 \mathrm{~min}$, blocked with 5\% bovine serum albumin in PBS for $10 \mathrm{~min}$, permeabilized with $0.2 \%$ Triton X-100 in PBS for $5 \mathrm{~min}$, and incubated overnight with the antibodies. The secondary antibody (Cy3-AffiniPure Goat Anti-Rabbit IgG, Jackson ImmunoResearch) was added and cells were incubated at room temperature for $1 \mathrm{~h}$. Nuclei were stained by DAPI and sections were sealed by glycerinum. Observations were performed at 40x magnification. Images were captured with a Zeiss confocal microscope and analyzed by LSM510 and Image J software (National Institutes of Health (NIH), Bethesda, MD).

\section{Western blot}

RIPA Lysis buffer was added to intestinal tissues ( $1 \mathrm{~mL}$ lysate for $1 \mathrm{~mL}$ tissue) and HUVECs on ice for 1 $\mathrm{h}$ and centrifuged at $1000 \mathrm{~g}$ for $15 \mathrm{~min}$. Supernatants were harvested and the protein concentrations were measured using a BCA Kit. Thirty micrograms of protein from each sample was used for SDS-PAGE and blocked by 5\% BSA sealing fluid for $1 \mathrm{~h}$ at room temperature. Primary antibodies were added: JAM-C (1:200; Santa Cruz Biotechnology), AQP-1 (1:1000; Abcam), MMP9 (1:1000; Abcam), and GAPDH (1:1000; Abcam) for $1 \mathrm{~h}$ at room temperature followed by horseradish peroxidase-coupled secondary antibody (1:5000; Jackson ImmunoResearch) for $1 \mathrm{~h}$ at room temperature. After $4{ }^{\circ} \mathrm{C}$ incubation overnight and 10 min washing with Tris-buffered saline Tween buffer three times, ECL substrate was added and imaging was obtained by using Bio-rad Gel Doc EZ gel imaging system. Gre value analysis of the target bars was performed by using Image J software (NIH).

Reverse transcription-polymerase chain reaction (RT-PCR)

Total RNA was extracted from the intestinal tissues and HUVECs with TRIzol reagent (Invitrogen). Polymerase chain reaction (PCR) primers were designed to be isoform specific. Following the instructions of the ReverTra Ace qPCR RT Kit (Toyobo), complementary DNA was generated from 1- $\mu$ g purified total RNA by reverse transcription $\left(30 \mathrm{~min}\right.$ at $37^{\circ} \mathrm{C}$ and $5 \mathrm{~min}$ at $\left.98^{\circ} \mathrm{C}\right)$.

To quantify the expression of the messenger RNA (mRNA) of AQP-1, MMP9 and JAM-C, a semiquantitative PCR system was set up using $2 \mu \mathrm{L}$ of $10 \mathrm{x}$ PCR Buffer, $1 \mu \mathrm{L}$ of dNTP at $2.5 \mathrm{mM}, 1 \mu \mathrm{L}$ of $\mathrm{MgCl}_{2}$ at $25 \mathrm{mM}, 1$ $\mu \mathrm{L}$ of forward and reverse primers at $10 \mu \mathrm{mol} / \mathrm{l}, 1 \mu \mathrm{L}$ of complementary DNA, $0.25 \mu \mathrm{L}$ of Taq enzyme, and deionized water to a total volume of $20 \mu \mathrm{L}$. The thermal cycle for amplification was as follows: (1) $94{ }^{\circ} \mathrm{C}$ for $3 \mathrm{~min}$; (2) 28 cycles of denaturation, each consisting of $30 \mathrm{~s}$ at $94{ }^{\circ} \mathrm{C}, 45 \mathrm{~s}$ at $58{ }^{\circ} \mathrm{C}$, and $20 \mathrm{~s}$ at $72{ }^{\circ} \mathrm{C}$ followed by (3) a final extension for $7 \mathrm{~min}$ at $70^{\circ} \mathrm{C}$. GAPDH amplification sequences were used as an internal control. PCR products were subjected to electrophoresis in 1.5\% agarose gel.

The sequences of PCR primers used were as follows:

HUVECs: AQP-1 (forward) 5'-CCTCCTGGCTATTGACTACA-3', (reverse) 5'-CCAGGATGAA-GTCGTAGATG-3';

MMP-9 (forward) 5'-TCGTCATCCAGTTTGGTGTC-3', (reverse) 5'-ACAA-CTCGTCATCGTCGAAA-3';

JAM-C (forward) 5'-CAGTAGGCAAGATGGCAACA-3', (reverse) 5'-CATCATTGCGATACCAGCTG-3';

GAPDH(forward) 5'-CTGCCAACGTGTCAGTG-GTG-3', 


\section{Cellular Physiology Cell Physiol Biochem 2017;44:2395-2406 \\ \begin{tabular}{l|l|l} 
DOI: 10.1159/000486155 & $\begin{array}{l}\text { O 2017 The Author(s). Published by S. Karger AG, Basel } \\
\text { www.karger.com/cpb }\end{array}$ \\
\hline
\end{tabular} \\ Pan et al:: Dachengqi Decoction Ameliorates Intestinal Injury in Experimental Acute}

Pancreatitis

(reverse) 5'-AAAGGCCAACAGCACAGTCA-3'.

Rats: AQP-1 (forward) 5'-TTGCCATTGGCTTGTCTGTG-3',

(reverse) 5'-ATGAATGGTCCC-ACCCAGAA-3';

MMP-9(forward) $5^{\prime}$-CTTTCTTATTGCCCGCACGT-3',

(reverse) 5'-ACATTTT-GCGCCCAGAGAAG-3';

JAM-C(forward) 5'-TGCAGAGTTCCAAAGGCTGT-3',

(reverse) 5'-ACATCATTGCGGTACCAGCT-3';

GAPDH (forward) 5'-GGCAAGTTCAACGGCACAGT-3',

(reverse) 5'- ATGACATACTCAGCACCGGC-3'.

\section{Statistical analysis}

All experiments were repeated at least three times. Values are given as means \pm SEM. Data were analyzed using SPSS 18.0 software. Statistical significance was assessed by one-way ANOVA. $P$ values less than 0.05 were considered significant.

\section{Results}

\section{Damaged HUVECs model established by TNF- $\alpha$ stimulation}

HUVECs were treated with $10 \mathrm{ng} / \mathrm{mL}$ of TNF- $\alpha$ for $6 \mathrm{~h}, 12 \mathrm{~h}$, and $24 \mathrm{~h}$. It is known that TNF- $\alpha$ promotes cell apoptosis. In this study, we used HUVECs to explore the role of TNF- $\alpha$ on apoptosis in vascular endothelial cells. The flow cytometry results showed that the apoptosis rate of HUVECs was increased in the TNF- $\alpha$ group (Fig. 1A). These results suggested TNF- $\alpha$ can induce apoptosis in HUVECs. The cell permeability of the TNF- $\alpha$ group was higher than that of the control group at different time points $(P<0.01)$. Permeability was higher with longer incubation times $(P<0.05$; Fig. 1B).

We explored the role of endothelial barrier function-associated proteins AQP-1, MMP9, and JAM-C in TNF- $\alpha$-induced damaged HUVECs, with RT-PCR, immunocytofluorescence, and western blot assays. As shown in Fig. $1 \mathrm{C}$, D, and E, the expression of AQP-1 protein was lower in the TNF- $\alpha$ group than in the control group at $12 \mathrm{~h}$, but MMP9 and JAM-C proteins had higher expression $(P<0.05)$. AQP-1 mRNA expression was lower in the TNF- $\alpha$ group than in the control group at $12 \mathrm{~h}$, and both MMP9 and JAM-C mRNA levels were higher $(P<0.05)$.

\section{Effects of DCQD on the damaged HUVEC model}

We used CCK-8 assay to find the optimal concentration of DCQD for the HUVECs. When the concentration of DCQD was in the range $0.0001-100 \mu \mathrm{g} / \mathrm{mL}$ at $6 \mathrm{~h}$, the survival rate of the HUVECs barely changed. However, at $12 \mathrm{~h}$ and $24 \mathrm{~h}$, the survival rates increased with increasing concentrations of DCQD. When the concentration of DCQD was $1 \mathrm{mg} / \mathrm{mL}$, the survival rates at $6 \mathrm{~h}, 12 \mathrm{~h}$, and $24 \mathrm{~h}$ fell sharply. Thus, we chose $100 \mu \mathrm{g} / \mathrm{mL}$ as the optimal concentration of DCQD (Fig.2A).

Cell permeabilities in the TNF- $\alpha$ group, Ang- 1 group, and DCQD group were higher than those in the control group at different time points $(P<0.01)$. The permeabilities in the Ang-1 and DCQD groups were much lower at the beginning of the $6 \mathrm{~h}$ and were lower after longer incubation times in comparison with the TNF- $\alpha$ group $(P<0.05$; Fig. $2 \mathrm{~B})$.

The apoptosis rate of the HUVECs in the TNF- $\alpha$ group was higher than that in the control group, while the rates were lower in the DCQD and Ang-1 groups than in the TNF- $\alpha$ group $(P$ $<0.05$; Fig. 2C). These results suggest that DCQD can reduce apoptosis in HUVECs.

Effects of DCQD on the expression of AQP-1, MMP9, and JAM-C in the damaged HUVECS model

The expression of AQP-1 mRNA was lower in the TNF- $\alpha$, Ang-1, and DCQD groups than in the control group at $12 \mathrm{~h}$, while the levels of MMP9 and JAM-C mRNA were higher $(P<$ 0.05; Fig. 3A). However, the mRNA expression of the AQP-1 gene in the DCQD group was 
Pan et al.: Dachengqi Decoction Ameliorates Intestinal Injury in Experimental Acute Pancreatitis

Fig. 1. Damaged HUVECs model established by TNF- $\alpha$ treatment. A. Apoptosis of HUVECs treated with $0 \mathrm{ng} / \mathrm{mL}$ TNF- $\alpha$ and $10 \mathrm{ng} / \mathrm{mL}$ TNF- $\alpha$ DMEM for $6 \mathrm{~h}, 12 \mathrm{~h}$, and $24 \mathrm{~h}$. B. Permeability of HUVECs in the TNF- $\alpha$ group was much higher than that of the control group. Effects of TNF- $\alpha$ on expression of AQP-1, JAM-C, and MMP9. C. mRNA expression of AQP-1, JAM-C, and MMP9 in HUVECs treated with $0 \mathrm{ng} / \mathrm{mL}$ and $10 \mathrm{ng} / \mathrm{mL}$ TNF- $\alpha$ for $12 \mathrm{~h}$ by RT-PCR. D. Expression of AQP-1, JAM-C and MMP9 proteins in HUVECs treated with $0 \mathrm{ng} / \mathrm{mL}$ and $10 \mathrm{ng} / \mathrm{mL}$ TNF- $\alpha$ for $12 \mathrm{~h}$ by western blot. E: Expression of AQP-1, JAM-C, and MMP9 in HUVECs treated with $0 \mathrm{ng} / \mathrm{mL}$ and $10 \mathrm{ng} / \mathrm{mL}$ TNF- $\alpha$ respectively for 12 $\mathrm{h}$ by immunocytofluorescence. ${ }^{*} \mathrm{P}<0.05$, ${ }^{* *} \mathrm{P}<0.01$, compared with the control group. Ang-1 denotes the TNF- $\alpha$ plus Ang-1 group, DCQD denotes the TNF- $\alpha$ plus DCQD group.

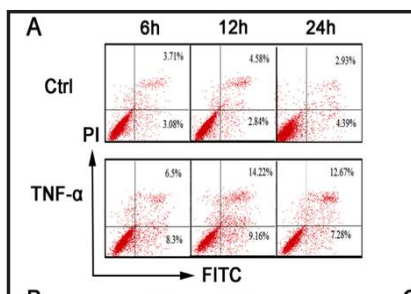

B

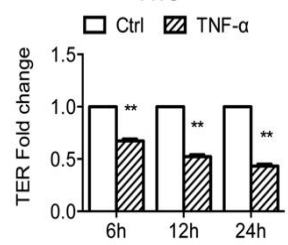

D

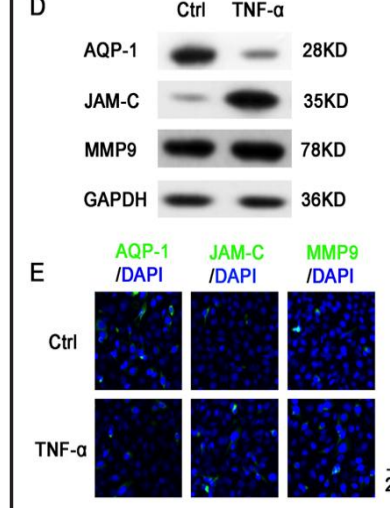

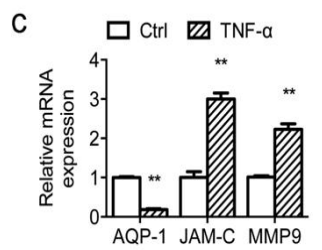
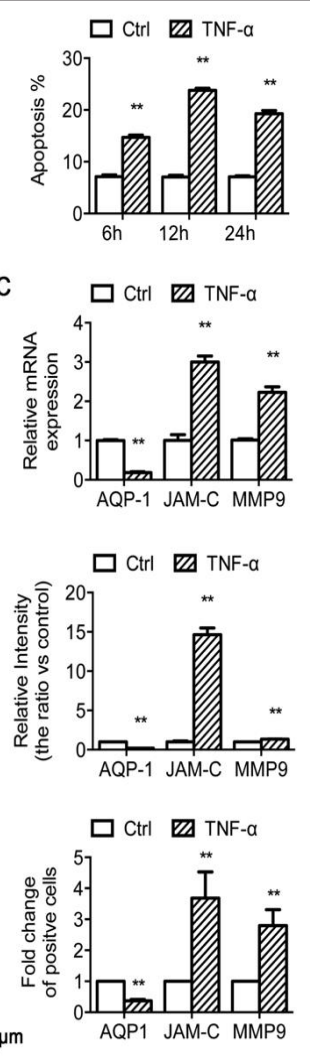

Fig. 2. $D C Q D$ reduced the level of damage in TNF- $\alpha$ treated HUVECs. A. CCK-8 assay showing that $100 \mu \mathrm{g} /$ $\mathrm{mL}$ DCQD was the appropriate dose. B. Permeability of HUVECs in the DCQD group was much lower than that in the TNF- $\alpha$ group. C. Apoptosis in HUVECs treated with DMEM, $10 \mathrm{ng} /$ $\mathrm{mL}$ TNF- $\alpha, 100$

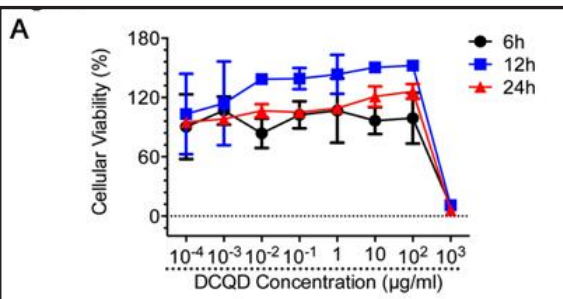

B
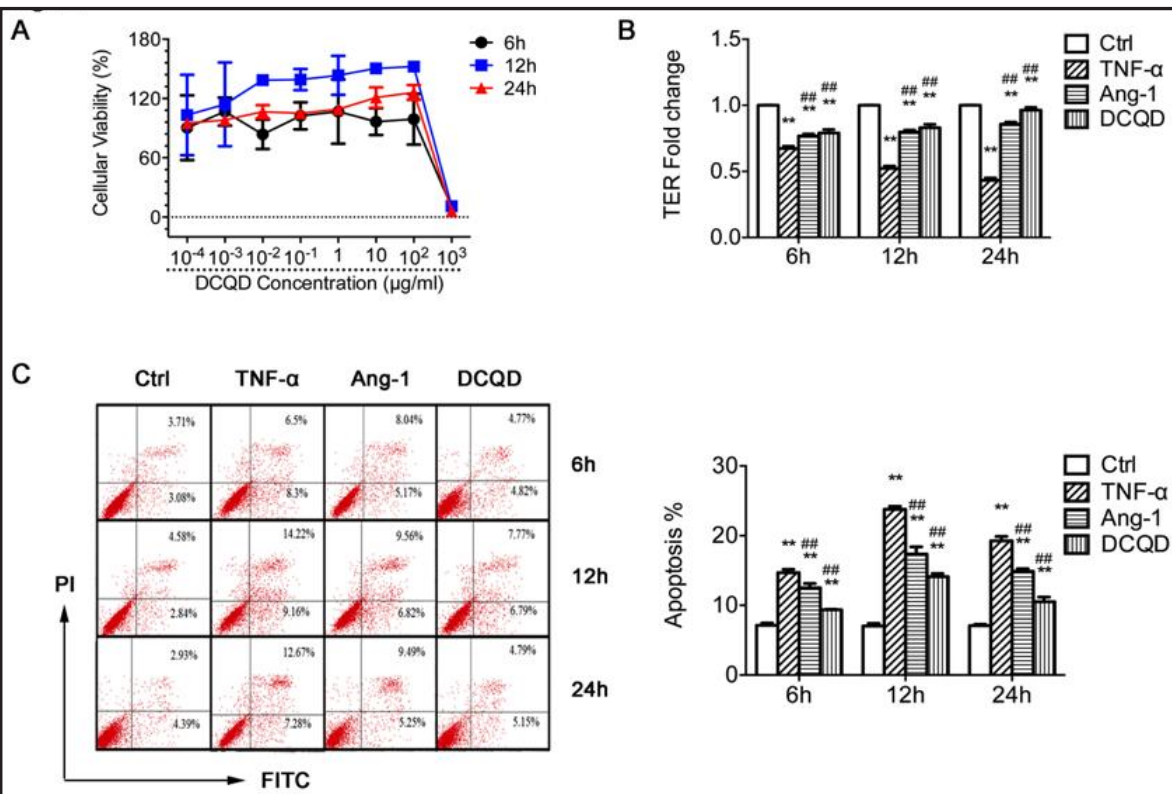

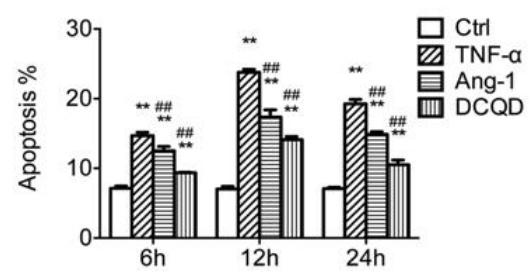

ng/mL Ang-1,

and $100 \mu \mathrm{g} / \mathrm{mL}$ DCQD for $6 \mathrm{~h}, 12 \mathrm{~h}$ and $24 \mathrm{~h} .{ }^{*} \mathrm{P}<0.05,{ }^{* *} \mathrm{P}<0.01$, compared with the control group; ${ }^{\#} \mathrm{P}<0.05$, $\#$ \# $\mathrm{P}<0.01$, compared with the TNF- $\alpha$ group. Ang- 1 denotes the TNF- $\alpha$ plus Ang-1 group, DCQD denotes the TNF- $\alpha$ plus DCQD group.

higher than that in the TNF- $\alpha$ group, while MMP9 and JAM-C mRNA expression levels were lower than those in the TNF- $\alpha$ group $(P<0.05)$. 
Fig. 3. DCQD regulates AQP-1, MMP9, and JAM-C and protects against damage in the HUVECs model. A. Expression of AQP-1, JAM-C, and MMP9 in HUVECs treated with DMEM, $10 \mathrm{ng} / \mathrm{mL}$ TNF- $\alpha, 100$ ng/mL Ang-1, and 100 $\mu \mathrm{g} / \mathrm{mL}$ DCQD for $12 \mathrm{~h}$ by RT-PCR. B. Expression of AQP-1, JAM-C, and MMP9 in HUVECs treated with DMEM, 10 ng/mL TNF- $\alpha, 100$ ng/ $\mathrm{mL}$ Ang-1, and $100 \mu \mathrm{g} / \mathrm{mL}$ DCQD for $12 \mathrm{~h}$ by western blot. C: Expression of AQP-1, JAM-C, and MMP9 in HUVECs treated with $10 \mathrm{ng} /$ mL TNF- $\alpha, 100 \mathrm{ng} / \mathrm{mL}$ Ang-1, and $100 \mu \mathrm{g} / \mathrm{mL}$ DCQD by immunocytofluorescence. ${ }^{*} \mathrm{P}<0.05,{ }^{* *} \mathrm{P}<0.01$, compared with the control group; ${ }^{\#} \mathrm{P}<0.05$, ${ }^{\# \#} \mathrm{P}<0.01$, compared with the TNF- $\alpha$ group. Ang-1 denotes the TNF- $\alpha$ plus Ang-1 group; DCQD denotes the TNF- $\alpha$ plus DCQD group.

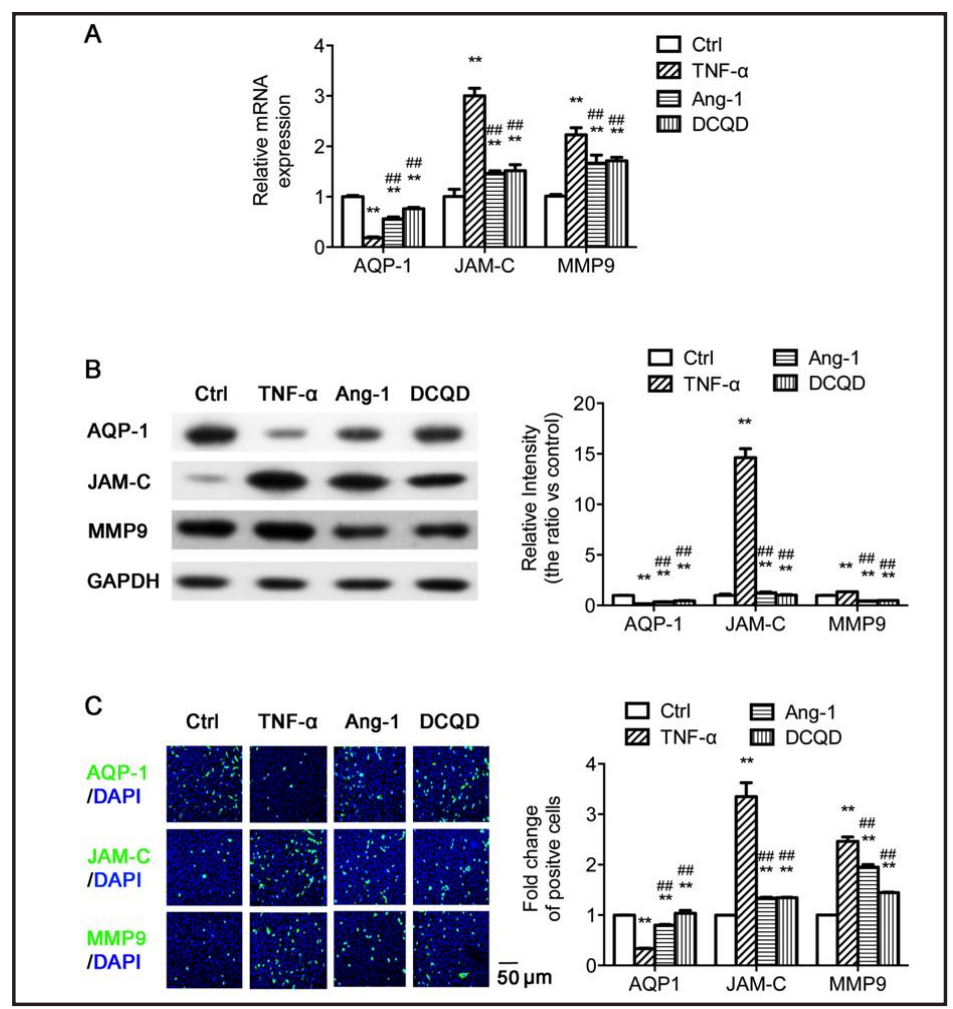

The expression of AQP-1 protein was lower in the TNF- $\alpha$, Ang-1, and DCQD groups than in the control group at $12 \mathrm{~h}$, but the expression of MMP9 and JAM-C proteins was higher $(P$ $<0.05$ ) Expression of AQP-1 protein was higher in the DCQD group than in the TNF- $\alpha$ group, and MMP9 and JAM-C levels were lower $(P<0.05)$ (Fig. 3 B and C).

Effects of DCQT on serum levels of inflammatory cytokines and intestinal injury in SAP rats

From these results, we found that DCQD reduced the damage to HUVECs caused by TNF- $\alpha$ and altered the level of expression of AQP-1, MMP9, and JAM-C. We used a rat model to verify the action of DCQD in intestinal injury caused by SAP. The morphological changes in the intestine are shown in Fig. 4A. The intestinal tissue of the rats in the control group showed no obvious changes but that in the SAP group was pathologically damaged. Erythrocytes and inflammatory cells were observed in the interstitium of the intestine and the Chiu's score of the SAP group was much higher than that of the control group. Moreover, the scores of the Ang- 1 and DCQD groups were much better than that of the SAP group, $(P<0.01$; Fig. 4A). The capillary permeability of the intestinal tissue in the SAP group was significantly higher than that of the control group $(P<0.01)$, and that of the Ang- 1 and DCQD groups $(P<0.01$; Fig. 4B).

The serum levels of amylase, TNF- $\alpha$, IL-1 $\beta$, IL-2, and IL- 6 from representative rats are shown in Fig. 4. The serum levels of amylase, TNF- $\alpha$, IL-1 $\beta$, IL-2 and IL- 6 were higher in the SAP group than in the control group $(P<0.05)$ and DCQD group $(P<0.05 ;$ Fig. 4 C, D, E, F, and G).

Effects of DCQD on the expression of AQP-1, MMP9 and JAM-C in intestinal tissue of SAP rats

RT-PCR results showed that the expression levels of AQP-1 mRNA were significantly lower in the SAP group than in the control group, while the expression levels of JAM-C and MMP9 were significantly increased $(P<0.01)$. AQP-1 mRNA expression levels in the intestinal tissue of the Ang-1 and DCQD groups were higher than in the SAP group, while JAM-C and MMP9 mRNA levels were lower $(P<0.01$; Fig. 5A). 
Fig. 4. DCQD improves SAP in LPS-cerulein-induced SAP rats. A. Pathological changes (HE) and pathological scores of intestinal tissue sections. B. Capillary permeability of intestine tissue in control, SAP, Ang-1, and DCQD groups by Evans blue extravasation. C-G. Levels of amylase, TNF- $\alpha$, IL- $1 \beta$, IL-2, and IL-6 in serum of rats in the control group, SAP, Ang-1, and DCQD groups. ${ }^{*} \mathrm{P}<0.05,{ }^{* *} \mathrm{P}<0.01$, compared with the control group; ${ }^{\#} \mathrm{P}<0.05$, \#\# $\mathrm{P}<0.01, \quad$ compared with the SAP group. $n=10$. Ang-1 denotes the SAP plus Ang-1 group, DCQD denotes the SAP plus DCQD group.

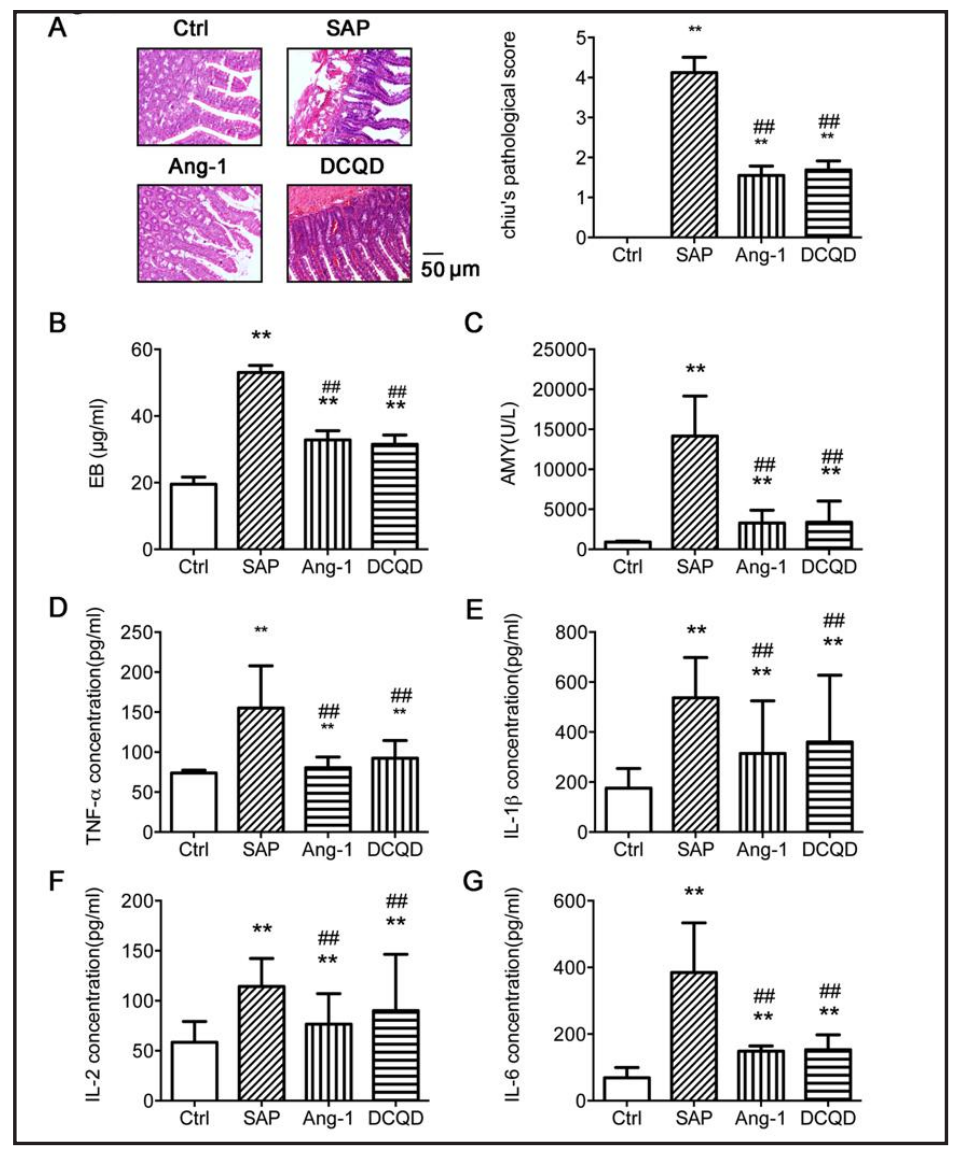

Fig. 5. DCQD regulates AQP-1, MMP9 and JAM-C to protect LPScerulein-induced SAP rats.A. mRNA expression of AQP-1, MMP9, and JAM-C translocation after DCQD treatment by RT-PCR. B. Protein expression of AQP-1, MMP9, and JAM-C in intestinal tissues by western blot. C. Protein expression of AQP-1, MMP9, and JAM-C in intestinal tissues by immunofluorescence. $\quad{ }^{*} \mathrm{P}<0.05,{ }^{* *} \mathrm{P}<0.01$, compared with the control group; ${ }^{\#} \mathrm{P}<0.05$, \# $\mathrm{P}<0.01, \quad$ compared with the SAP group. $n=10$. Ang-1 denotes the SAP plus Ang-1 group, DCQD denotes the SAP plus DCQD group.

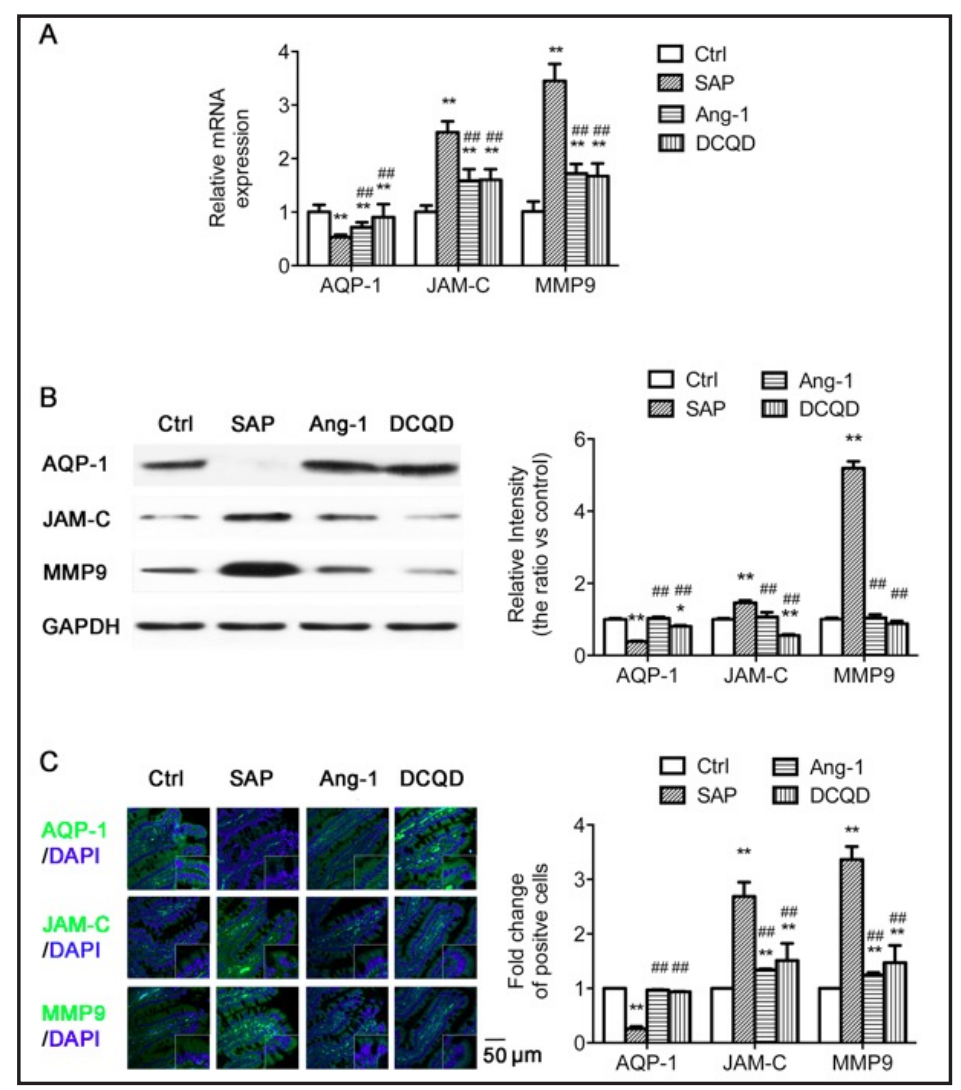


To determine the expression and cellular localization of AQP-1, MMP9, and JAM-C in intestinal tissue, immunohistofluorescence and western blot assays were performed (Fig. 5 $B$ and C). The expression of AQP-1 protein in the intestinal tissue of the SAP group was lower than in the control group while JAM-C and MMP9 protein expression levels were higher $(P$ $<0.01$ ). The expression of AQP-1 protein in the intestinal tissues of the Ang-1 and DCQD groups was higher than in the SAP group, while JAM-C and MMP9 protein levels were lower $(P<0.01$; Fig. 5 B and C).

\section{Discussion}

TNF- $\alpha$ is an important factor in the inflammatory reaction processes of the human body. TNF- $\alpha$ receptor exists on the surface of vascular endothelial cells. Studies showed that abnormally high levels of TNF- $\alpha$ in the blood damage the vascular endothelial cells and promote the expression of endothelial cell adhesion molecules to strengthen the adhesion to neutrophils. Moreover, TNF- $\alpha$ promotes endothelial cells to produce more inflammatory factors, which further increases the vascular permeability and inflammatory reactions [1921]. Hence, it is often used to study the mechanisms of endothelial cell injury in vitro.

In this study, we used TNF- $\alpha$ to establish a cell injury model in HUVECs. We observed changes in permeability and apoptosis rates in endothelial cells. Since AQP-1, MMP9, and JAM-C all play key roles in regulating capillary permeability in SAP [9-11], we also observed changes in the expression of endothelial barrier function-related proteins such as AQP-1, MMP-9, and JAM-C proteins and mRNA. We found that after adding TNF- $\alpha(10 \mathrm{ng} / \mathrm{mL})$, the TEER permeability measurements and apoptosis rates of the endothelial cells increased at $6 \mathrm{~h}, 12 \mathrm{~h}$, and $24 \mathrm{~h}$. These results are consistent with the results of our previous study [22]. The most significant rise in the rate of apoptosis took place at $12 \mathrm{~h}$, so we chose $12 \mathrm{~h}$ as a check point. We found that the expression of AQP-1 protein decreased, while the expression of both MMP-9 and JAM-C increased in the TNF- $\alpha$ group compared with the control group. The mRNA expression of each protein changed correspondingly. This demonstrated that changes in the endothelial cell membrane-related proteins indeed influenced endothelial barrier function in SAP.

In recent years, research into Angiopoietin has substantially altered our understanding of the pathological process of capillary leak syndrome in SAP. Ang comprises a family of specific endothelial cells secrete growth factors. Ang- 1 is the only endogenous protein factor that can prevent the damage of vascular leakage among the angiogenic factors family. Ang-1 promotes endothelial cell chemotaxis and aggregation, and inhibits apoptosis, inflammation, seepage, and leukocyte adhesion. It can also regulate the proliferation of endothelial cells and vascular smooth muscle cells to promote the maturation of blood vessels, which is very important for maintaining the stability and integrity of blood vessels [23-25]. Our previous studies found that serum Ang-1 levels in AP rats are significantly decreased, and after treatment with Ang-1, the amounts of inflammatory factors in serum dramatically decline and the capillary permeability of tissue decreases [26]. Therefore, this experiment used Ang1 in the positive control group.

With the human recombinant Ang-1 and DCQD freeze-dried powder intervention, we found that the permeability and apoptosis rates of the endothelial cells decreased. Compared with the TNF- $\alpha$ group, the expression of AQP-1 protein and mRNA increased and the expression of MMP-9 and JAM-C decreased. These results showed that DCQD could effectively alleviate the capillary leakage and the damage to the endothelial barrier function due to the inflammatory reaction process via regulation of the endothelial barrier-related proteins. The results were consistent with the results of our previous study $[9,26]$.

To verify the results, we established a SAP rat model by intraperitoneal injection with cerulein plus lipopolysaccharides (LPS), intervened by tail vein injection with Ang-1 and gavage with DCQD. We subsequently sought to identify inflammatory factors in the serum and intestinal damage among the rats of each group. We also observed the correlation between 
endothelial barrier function-related proteins and intestinal capillary permeability. Our model was deemed successful. Serum amylase, capillary permeability, and the expression of inflammatory factors such as IL-2, IL-6, IL-1 $\beta$, and TNF- $\alpha$ appeared in the SAP group as previous research has shown [27].

However, compared with the SAP group, the pathological damage to the intestinal tissues of the Ang-1 and DCQD groups were significantly alleviated alongside obvious decreases in the levels of amylase in the serum, capillary permeability, and the expression of inflammatory factors, such as IL-2, IL-6, IL-1 $\beta$, and TNF- $\alpha$. These results showed that Ang1 and DCQD could effectively alleviate intestinal capillary leakage in rat models with acute pancreatitis, and might play a positive role in protecting the microcirculation of the intestine.

In addition, we detected changes in the expression of AQP-1, MMP-9, and JAM-C, the endothelial cell barrier function-related proteins, in rats intestinal tissues after the intervention with DCQD and Ang-1. Ang-1 and DCQD significantly upregulated the expression of AQP-1 protein and downregulated the expression of MMP-9 and JAM-C proteins. Thus, Ang-1 and DCQD alleviated intestinal injury in SAP, decreased capillary permeability in the intestinal mucosa, and reduced capillary leakage by regulating the vascular endothelial barrier function-related proteins.

In summary, acute gastrointestinal dysfunction is a severe complication of SAP. However, recovery of the intestinal capillary endothelial barrier dysfunction can alleviate intestinal damage in SAP. Hence, treatment of intestinal capillary endothelial barrier dysfunction is key to the treatment of SAP complicated by intestinal damage. DCQD not only relieves damage the capillaries due to cytokines and inflammatory factors by inhibiting the release of these factors, but also regulates capillary permeability and alleviates endothelial damage effectively by altering the expression of endothelial cells barrier proteins. Our findings may provide a new direction and therapeutic target for the prevention and treatment of SAPrelated intestinal injury.

\section{Acknowledgements}

This work was supported by the Medical Guide Program of Shanghai Committee of Science and Technology (14411972300), the Key Program by Shanghai Municipal Commission of Health and Family Planning (201440027), a General Program Grant from the National Natural Science Foundation of China (81673789), the Xinglin New Star Innovation Program (2016XL0807), and the Key Medical Discipline Project of Shanghai Putuo District. We thank Thinkscience.co for linguistic assistance during the preparation of this manuscript.

\section{Disclosure Statement}

No conflict of interest exists.

\section{References}

1 Yuan J, Pandol SJ: PKD signaling and pancreatitis. J Gastroenterol 2016;51:651-659.

2 Nesvaderani M, Eslick GD, Vagg D, Faraj S, Cox MR: Epidemiology, aetiology and outcomes of acute pancreatitis: A retrospective cohort study. Int J Surg 2015;23:68-74.

Thoeni RF: Imaging of Acute Pancreatitis. Radiol Clin North Am 2015;53:1189-1208.

Sah RP, Saluja A: Molecular mechanisms of pancreatic injury. Curr Opin Gastroenterol 2011;27:444-451.

Thrower EC, Gorelick FS, Husain SZ: Molecular and cellular mechanisms of pancreatic injury. Curr Opin Gastroenterol 2010;26:484-489.

Bumbasirevic V, Radenkovic D, Jankovic Z, Karamarkovic A, Jovanovic B, Milic N, Palibrk I, Ivancevic $\mathrm{N}$ : Severe acute pancreatitis: overall and early versus late mortality in intensive care units. Pancreas 2009;38:122-125. 


\section{Cellular Physiology Cell Physiol Biochem 2017;44:2395-2406 \begin{tabular}{l|l} 
and Biochemistry & DOI: 10.1159/000486155 2017 The Author(s). Published by S. Karger AG, Basel \\
(c)
\end{tabular}

Pan et al.: Dachengqi Decoction Ameliorates Intestinal Injury in Experimental Acute Pancreatitis

7 Zou XP, Chen M, Wei W, Cao J, Chen L, Tian M: Effects of enteral immunonutrition on the maintenance of gut barrier function and immune function in pigs with severe acute pancreatitis. JPEN J Parenter Enteral Nutr 2010;34:554-566.

8 Kylanpaa ML, Repo H, Puolakkainen PA: Inflammation and immunosuppression in severe acute pancreatitis. World J Gastroenterol 2010;16:2867-2872.

-9 Feng DX, Peng W, Chen YF, Chen T, Tian JY, Si HR, Cai J, Rao Y, Han F, Zhao R: Down-regulation of aquaporin 1 in rats with experimental acute necrotizing pancreatitis. Pancreas 2012;41:1092-1098.

10 Sochor M, Richter S, Schmidt A, Hempel S, Hopt UT, Keck T: Inhibition of matrix metalloproteinase-9 with doxycycline reduces pancreatitis-associated lung injury. Digestion 2009;80:65-73.

-11 Wu D, Zeng Y, Fan Y, Wu J, Mulatibieke T, Ni J, Yu G, Wan R, Wang X, Hu G: Reverse-migrated neutrophils regulated by JAM-C are involved in acute pancreatitis-associated lung injury. Sci Rep 2016;6:20545.

-12 Gong HL, Tang WF, Yu Q Xiang J, Xia Q, Chen GY, Huang X, Liang MZ: Effect of severe acute pancreatitis on pharmacokinetics of Da-Cheng-Qi Decoction components. World J Gastroenterol 2009;15:5992-5999.

13 Zhao J, Tang W, Wang J, Xiang J, Gong H, Chen G: Pharmacokinetic and pharmacodynamic studies of four major phytochemical components of Da-Cheng-Qi decoction to treat acute pancreatitis. J Pharmacol Sci 2013;122:118-127.

14 Ren YY, Gong HL, Tang WF, Wan MH, Zhao JL, Huang X: [Dachengqi Decoction induces pancreatic acinar cell apoptosis in experimental acute pancreatitis in rats]. Zhong Xi Yi Jie He Xue Bao 2009;7:651-656.

15 Chen Z, Chen Y, Pan L, Li H, Tu J, Liu C, Dai X, Zhang X, Sun G, Feng D: Dachengqi Decoction Attenuates Inflammatory Response via Inhibiting HMGB1 Mediated NF-kappaB and P38 MAPK Signaling Pathways in Severe Acute Pancreatitis. Cell Physiol Biochem 2015;37:1379-1389.

16 Szczepanski M, Kamianowska M, Wnuczko K, Pietruczuk M, Wolczynski S: [The influence of erythropoietin (Epo) on intercellular adhesion molecule-1 (ICAM-1, CD54) and platelet-endothelial cell adhesion molecule-1 (PECAM-1, CD31) expression on human umbilical vein endothelial cells (HUVEC) induced by tumor necrosis factor-alpha (TNF-alpha)]. Wiad Lek 2007;60:22-27.

17 Chiu CJ, McArdle AH, Brown R, Scott HJ, Gurd FN: Intestinal mucosal lesion in low-flow states. I. A morphological, hemodynamic, and metabolic reappraisal. Arch Surg 1970;101:478-483.

18 Moitra J, Sammani S, Garcia JG: Re-evaluation of Evans Blue dye as a marker of albumin clearance in murine models of acute lung injury. Transl Res 2007;150:253-265.

19 Fu BD, Yamawaki H, Okada M, Hara Y: Vaspin can not inhibit TNF-alpha-induced inflammation of human umbilical vein endothelial cells. J Vet Med Sci 2009;71:1201-1207.

20 Chen HW, Lin AH, Chu HC, Li CC, Tsai CW, Chao CY, Wang CJ, Lii CK, Liu KL: Inhibition of TNF-alpha-Induced Inflammation by andrographolide via down-regulation of the PI3K/Akt signaling pathway. J Nat Prod 2011;74:2408-2413.

-21 Liu X, Pan L, Wang X, Gong Q, Zhu YZ: Leonurine protects against tumor necrosis factor-alpha-mediated inflammation in human umbilical vein endothelial cells. Atherosclerosis 2012;222:34-42.

22 Jiang C, Fang X, Jiang Y, Shen F, Hu Z, Li X, Huang X: TNF-alpha induces vascular endothelial cells apoptosis through overexpressing pregnancy induced noncoding RNA in Kawasaki disease model. Int J Biochem Cell Biol 2016;72:118-124.

-23 Alfieri A, Ong AC, Kammerer RA, Solanky T, Bate S, Tasab M, Brown NJ, Brookes ZL: Angiopoietin-1 regulates microvascular reactivity and protects the microcirculation during acute endothelial dysfunction: role of eNOS and VE-cadherin. Pharmacol Res 2014;80:43-51.

24 Kim S, Kwon J: COMP-Ang1 inhibits apoptosis as well as improves the attenuated osteogenic differentiation of mesenchymal stem cells induced by advanced glycation end products. Biochim Biophys Acta 2013;1830:4928-4934.

-25 Ni J, Verbavatz JM, Rippe A, Boisde I, Moulin P, Rippe B, Verkman AS, Devuyst O: Aquaporin-1 plays an essential role in water permeability and ultrafiltration during peritoneal dialysis. Kidney Int 2006;69:15181525.

26 Chen YF, Kong PT, Li HC, Fan XJ, Tu JM, Xie JK, Tian JY, Pan LY, Chen T, Cao YJ, Yin PH, Peng W, Feng DX: Cartilage Oligomeric Matrix Protein-Angiopoietin-1 Has a Protective Effect of Vascular Endothelial Barrier in Rat With Acute Necrotizing Pancreatitis. Pancreas 2016;45:142-147.

27 Ding SP, Li JC, Jin C: A mouse model of severe acute pancreatitis induced with caerulein and lipopolysaccharide. World J Gastroenterol 2003;9:584-589. 